\title{
Cognitive Performance in Relapsing- Remitting Multiple Sclerosis: At Risk or Impaired?
}

\author{
Marta Altieri ${ }^{a, b, c}$ Mariangela Fratino ${ }^{a, b} \quad$ Ilaria Maestrini ${ }^{a, c}$ Claudia Dionisi ${ }^{a, b}$ \\ Rosanna Annecca ${ }^{a}$ b Edoardo Vicenzini ${ }^{a}$ Vittorio Di Piero ${ }^{a, c}$

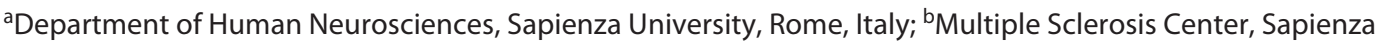 \\ University, Rome, Italy; ' Sub-Intensive Unit of Neurology, Sapienza University, Rome, Italy
}

\section{Keywords}

Multiple sclerosis · Cognition - Brief International Cognitive Assessment for Multiple Sclerosis · Cognitive impairment
0.03). Conclusions: $\mathrm{Cl}$ is a significant issue in MS and integration of a screening test, such as the SDMT, into routine clinical practice could be of worth to identify "at risk" patients and to promote an early therapeutic intervention.

(c) 2021 S. Karger AG, Base

\section{Introduction}

Cognitive impairment (CI) occurs in about 45\% (ranging from 20 up to $70 \%$ ) of multiple sclerosis (MS) patients, even in the early stages [1-3]. The variability in CI prevalence in MS patients relies on many factors such as different diagnostic criteria and neuropsychological batteries (NBs) used, clinical heterogeneity of MS population, and time interval from relapses. Many studies aimed at identifying specific neuropsychological and neuroradiological pattern profiles peculiar to different MS subtypes gave heterogeneous results [3-5].

In the DSM-5 criteria, since overt dementia is rare, the CI in MS might be classified as a "mild neurocognitive disorder" defined as modest cognitive decline in 1 or more cognitive domains (complex attention, executive function, learning and memory, language, perceptual- karger@karger.com

www.karger.com/dem

C 2021 S. Karger AG, Base

Karger"
Marta Altieri

Dipartimento di Neuroscienza Umane, Policlinico Umberto I Sapienza Università di Roma Viale dell'Università 30 IT-00185 Rome (Italy) centrosmaltieri@gmail.com 
motor, or social cognition). According to guidelines, test performance in mild neurocognitive disorder should fall in the range of 1-2 standard deviation (SD) below the normative mean, or between the $3 \mathrm{rd}$ and 16 th percentiles, on tests for which appropriate norms are available. Unfortunately, the DSM-5 does not specify which tests, or how many, should be administered per cognitive domain [6].

In MS, the neuropsychological assessment may show deficits of mental processing speed, memory, and attention [2]. In 2012, a panel of experts validated a brief cognitive tool that could be easily integrated in the clinical practice: the Brief International Cognitive Assessment for Multiple Sclerosis (BICAMS) [7]. It represents the first internationally validated and standardized cognitive measure for MS. BICAMS consists in Symbol Digit Modalities Test (SDMT), California Verbal Learning TestSecond Edition (CVLT-II), and the Brief Visuospatial Memory Test (BVMT-R). This brief cognitive tool could also be integrated into more detailed cognitive assessments. While the BICAMS benefits from ease of administration and could be administered by individuals who may not have neuropsychological training, the scoring is burdensome, time consuming, and leaves room for error. Two different cutoffs have been identified to simplify BICAMS interpretation: a more sensitive but less specific cutoff of $1.5 \mathrm{SD}$ below the mean, and a less sensitive but more specific cutoff of 2 SD below the mean [8].

In recent years, the $\mathrm{CI}$ in $\mathrm{MS}$ is shaping up as a dynamic phenomenon in which the disorder can not only worsen over time, but also improve or even regress $[1,9]$. In this view, it becomes necessary to identify patients at risk of CI as soon as possible, in order to apply therapeutic and rehabilitative strategies.

The aim of the present study was to evaluate the prevalence and predictors for $\mathrm{CI}$ in a population of consecutive mild relapsing-remitting (RR) MS patients using the BICAMS as a screening test. Patients scoring below the established score (2 SDs below the mean) in at least 1 test of the BICAMS were defined "at risk" for CI and underwent an extensive NB. Differences in NB subtest performances between "at risk" and CI patients were recorded.

\section{Materials and Methods}

During a 1-year period, according to the 2010 revised McDonald criteria [10], 82 consecutive RRMS outpatients were enrolled at the MS Center of "Sapienza" University of Rome. All MS patients were on first- or second-line treatment.
The inclusion criteria were as follows: Expanded Disability Status Scale (EDSS) score $\leq 3.5$ at screening; no evidence of relapse within 30 days prior to enrollment; $18-59$ years of age; and fluent in Italian. The exclusion criteria were as follows: neurological, psychiatric, or medical conditions besides MS and depression that might affect cognition; a history of learning disabilities; seizures; uncorrected visual acuity problems; corticosteroid treatment within 2 months of enrollment; current MS exacerbation; and current use of legal or illegal drugs that could impact on cognitive functions.

Patients were evaluated by a senior neurologist (M.A.) in order to attribute the EDSS score. The neuropsychological assessments started with the BICAMS [7] which is a screening test lasting about $15 \mathrm{~min}$ and evaluating information processing speed, verbal, and short-term visuospatial memory. Patients having $\geq 1$ altered test at the BICAMS underwent extensive NB examination including Stroop Word-Color interference Test [11] to measure selective attention, cognitive flexibility information, cognitive inhibition, Trail Making Test (TMT) [12] to measure executive functions and information processing speed, Rey-Osterrieth Complex Figure Test [13] to investigate constructional praxis, visuographic memory, and some aspects of planning and executive functions, with immediate and delayed recall, Phonemic (PVT) and Semantic Verbal Fluency (SVT) [14] and Aachener Aphasia Test (AAT) [15] to assess the lexical stock and the ability to access to the lexicon, and Weight and Time Estimation Test [16] to assess the ability to evaluate abstraction skills. NB assessment was performed by a neuropsychologist (M.F.) in a quiet room. The scores were adjusted for age, sex, and instruction levels as required to the normative standards based on the relative Italian validation manuals [17]. The research was conducted ethically in accordance with the World Medical Association Declaration of Helsinki and written informed consent was obtained.

\section{Statistical Analysis}

All statistical analyses were conducted using SPSS software version 25 (IBM Corp., Armonk, NY, USA). We first calculated zscores for all measures, based on normative data published for the Italian population.

According to the literature, CI was diagnosed if patients' performance was below $2 \mathrm{SD}$ of the mean of normative values in at least 2 different cognitive domains [2]. Patients scoring 2 SD below the mean at 1 test of the BICAMS were defined "at risk" for CI. Differences between "at risk" and CI patients were explored with the $\chi^{2}$ test with a correction for multiple comparison. $p$ values $<0.05$ were considered significant.

\section{Results}

We enrolled 82 RRMS consecutive patients (mean age $45.8 \pm 11,56 \mathrm{~F}$ ). Mean years of education was 15.2 years, and the mean EDSS score was $2.8( \pm 1.8)$. Average disease duration was 3.9 years. Demographic and clinical parameters of the entire population are shown in Table 1. The mean scores on the SDMT, CVLT-II, and BVMT-R were $36( \pm 9), 44( \pm 11)$, and $23( \pm 8.5)$, respectively. According
Altieri/Fratino/Maestrini/Dionisi/ Annecca/Vicenzini/Di Piero 
Table 1. Clinical and demographic parameters of the entire population

\begin{tabular}{lc}
\hline Age, mean (SD) & $45.8 \pm 11$ \\
Age $>40$ yrs & $57(69.5 \%)$ \\
Sex, $n(\%)$ & $56 \mathrm{~F}(68)$ \\
Education, mean (SD) & $13.2( \pm 3.2)$ \\
$\quad$ Education $>13$ yrs & $24(29.3 \%)$ \\
EDSS, mean (SD) & $2.8( \pm 1.8)$ \\
$\quad$ EDSS $>2.5$ & $35(42.7 \%)$ \\
Age at onset, mean (SD) & $37( \pm 11)$ \\
Disease duration, mean (SD) & $3.9 \pm 2$ \\
Relapses in $1 /$ yr & $6(7 \%)$ \\
\hline Disease-modifying treatments, n $(\%)$ & \\
Interferons & $10(12.2)$ \\
Galatiramer acetate & $4(4.8)$ \\
Dimethylfumarate & $20(24.3)$ \\
Teriflunomide & $17(20.7)$ \\
Fingolimod & $20(24.3)$ \\
Natalizumab & $9(11)$ \\
Alentuzumab & $2(2.4)$ \\
\hline
\end{tabular}

EDSS, Expanded Disability Status Scale.

to Beier and co-workers' criteria [8], 30 (36.6\%) patients had abnormal scores at the SDMT, 20 (24.4) at the BVMT$\mathrm{R}$, and $16(19.5 \%)$ at the CVLT-II.

Twenty-three percent of patients (19/82) having $\geq 2$ abnormal test score in at least 2 different cognitive domains were diagnosed as CI patient, and 25.6\% (21/82) having 1 abnormal score were defined "at risk" [18]. Patients having $\geq 1$ altered test at the BICAMS underwent extensive NB examination. At the univariate analysis, CI patients had more frequently an EDSS $\geq 2.5$, a higher number of relapses in the last 12 months, and were less educated in comparison to "at risk" patients. The differences between CI and "at risk" patients in neuropsychological assessment are shown in Table 2.

We grouped neuropsychological tests for the cognitive functions as follows: (1) executive functions: SDMT, TMT A and B, Stroop, PVF, STEP W, and STEP T, (2) memory: CVLT, BVMT, Rey Figure IR and DR and (3) language: AAT and SVF. We found that 20/21 "at risk" patients failed in executive functions, 11/21 in memory, and only $1 / 21$ in language tests. At the extensive NB evaluation, $16(76 \%)$ "at risk" patients had at least one other impaired test in another domain (i.e., a patient scoring below the mean in one BICAMS subtest must also have another pathological score in one of the domains mentioned above).

Cognition in MS
Table 2. Comparison of clinical and NP parameters between "at risk" and CI patients

\begin{tabular}{lccc}
\hline NP tests & $\begin{array}{c}\text { "At risk" } \\
(n=21)(\%)\end{array}$ & $\begin{array}{l}\text { CI } \\
(n=19)(\%)\end{array}$ & $p$ value \\
\hline Age $>40$ & $17(80)$ & $15(79)$ & ns \\
Sex F & $14(67)$ & $12(63)$ & ns \\
EDSS $>2.5$ & $9(43)$ & $14(74)$ & 0.05 \\
Education $>13$ yrs & $10(48)$ & $4(21)$ & 0.05 \\
Relapses in 1/yr & $1(5)$ & $3(15)$ & 0.03 \\
SDMT & $15(71)$ & $15(79)$ & ns \\
CVLT & $3(14.2)$ & $13(68.4)$ & $<0.001$ \\
BVMT & $3(14.2)$ & $16(84.2)$ & $<0.001$ \\
Stroop (T) & $4(19)$ & $8(42.1)$ & ns \\
TMT A & $5(3.8)$ & $15(78.9)$ & 0.001 \\
TMT B & $3(14.2)$ & $8(42.1)$ & 0.05 \\
Rey figure IR & 0 & $2(10.5)$ & ns \\
Rey figure DR & $6(28.5)$ & $8(42.1)$ & ns \\
PVF & $1(4.7)$ & $8(42.1)$ & 0.006 \\
SVF & $1(4.7)$ & $10(52.6)$ & 0.001 \\
AAT & 0 & 0 & ns \\
STEP W & $9(42.8)$ & $10(52.6)$ & ns \\
STEP T & $4(19)$ & $9(47.3)$ & 0.05 \\
\hline
\end{tabular}

CI: NP scores below 2 SD of the mean of normative values in $\geq 2$ different cognitive domains. "At risk": NP scores below 2 SD of the mean of normative values in 1 test. EDSS, Expanded Disability Status Scale; CI, cognitive impairment; SDMT, Symbol Digit Modalities Test; CVLT-II, California Verbal Learning Test-Second Edition; BVMT-R, Brief Visuospatial Memory Test; TMT, Trial Making Test; IR, Immediate Recall; DR, Delayed Recall; PVF, Phonemic Verbal Fluency; SVF, Semantic Verbal Fluency; AAT, Aachener Aphasia Test; STEP W, Weight Estimation Test; STEP $\mathrm{T}$, Time Estimation Test.

\section{Discussion}

This study shows that (i) about one-fourth of our mild RRMS patients were already cognitively impaired, (ii) about one-fourth were "at risk" of CI, having at least an abnormal score at cognitive tests, and (iii) about threefourths of "at risk" patients were already cognitively impaired at the extensive NB. These data are in accordance with recent investigations [19].

Despite advances in knowledge about the neural basis of cognitive function in MS, there are still uncertainties concerning what it is called "normal cognition." It follows that the assessment of cognitive dysfunction, typically defined as a performance below a chosen threshold in a number of cognitive domains, assessed in a specific neuropsychological test (e.g., 1.5-2 SD below normal of a zscore of one or more cognitive domains) is still a matter 
of debate. In these batteries, results are commonly expressed as "intact/preserved" or "impaired" [20], and numerous studies usually differ on CI definitions. For this reason, standardized batteries and diagnostic criteria are mandatory.

Another important issue concerns the need to identify patients at risk as soon as possible, in order to apply therapeutic and rehabilitative strategies. Recent studies evaluated the effect of disease-modifying therapies (DMTs) on cognitive deficits $[21,22]$. It is likely that the beneficial effects of DMTs on cognition arise from the prevention of inflammatory activity more than secondary neurodegeneration. In this view, early detection of patients at risk of cognitive decline could increase the benefit of DMTs in the inflammatory phase. Evaluation of cognitive effects beyond reduction of inflammatory activity would require a different trial's designs. They should specifically take into account the cognitive assessment, controlling for potential confounding effects, and adjusting for overall degree of alterations in structural and functional connectivity [20]. According to what is already know in literature, the SDMT, albeit with some limitations, seems to be the best psychometric measure available for screening $\mathrm{CI}$ in MS patients [23].

In our study, the SDMT was impaired in $70 \%$ of "at risk" patients and in $79 \%$ of those already impaired. This finding, though confirming the high sensitivity of the test, raises some doubts about its specificity [24]. A recent study showed that, though the application of the total BICAMS battery should be the goal to strive for, the tests showing the highest conformity with the total battery to diagnose CI were the SDMT and the BVMT-R [25]. In our sample, the BVMT-R was impaired in only 3 at-risk patients, but the combination of SDMT and BVMT was impaired in $>80 \%$ of CI patients. In this view, we can argue that processing speed could be the first cognitive function to be altered, and therefore influencing downstream processes such as learning, memory, word retrieval, and executive function [24]. This is in line with the recommendation of the National MS Society which indicates the SDMT as a baseline cognitive screening and an integral component of disease monitoring [26]. Following a positive screen with the SDMT, or other validated measure, a more thorough assessment by a neuropsychologist is recommended to assess areas of cognitive deficit and strength, as well as to evaluate all factors that could impact on cognitive functioning, such as cognitive reserve [27].

Considering predictors associated with CI, our results are in line with previous cross-sectional and longitudinal observations pointing to the association of CI with disability. Interestingly, this association was evident even though we chose patients with mild disability (EDSS $\leq$ 3.5) [26].

Clinical relapses, especially cortical ones, has been associated with CI in MS patients, as well as changes in SDMT and other neuroperformance scales [9]. However, it should be stressed that an improvement in cognitive conditions has also been reported. This was more evident the more temporally far from the relapse.

Our study has several limitations mainly represented by the small sample size and the lack of follow-up. Patients were classified as having CI when they failed at least 2 cognitive domains at the NP tests. This is in accordance with part of the literature, although others consider impaired patients failing in at least $3 \mathrm{NP}$ tests [28]. This discrepancy only emphasizes the need for standardized guidelines on the definition of CI in MS.

In conclusion, our results show that $\mathrm{CI}$ is a significant issue in MS and strongly support the need to integrate/incorporate a screening test, such as the SDMT, into routine clinical practice. This test has limited learning effects and is able to detect clinically meaningful change [29]. Moreover, it can easily help identify "at risk" patients, whom therefore request a complete NP evaluation. The importance of an assessment of cognitive function in patients diagnosed with RRMS could be fundamental to plan a therapeutic intervention or its early modification in order to preserve cognitive function.

Published in Celebration of the 30th Anniversary of the inception of Dementia and Geriatric Cognitive Disorders 1990-2020.

\section{Statement of Ethics}

The research was conducted ethically in accordance with the World Medical Association Declaration of Helsinki. Patients have given written informed consent.

\section{Conflict of Interest Statement}

Dr. Altieri is a board member of Dementia and Geriatric Cognitive Disorder. The authors have no conflicts of interest to declare.
Altieri/Fratino/Maestrini/Dionisi/ Annecca/Vicenzini/Di Piero 


\section{Funding Sources}

The authors did not receive any funding.

\section{Author Contributions}

Dr. Altieri, Dionisi, and Annecca selected, visited, and followed up MS patients involved in the study. Dr. Fratino and Altieri performed the MB assessment and wrote the paper. Dr. Maestrini, Vicenzini, and Di Piero reviewed manuscript and statistics.

\section{References}

1 Katsari M, Kasselimis DS, Giogkaraki E, Breza M, Evangelopoulos ME, Anagnostouli M, et al. A longitudinal study of cognitive function in multiple sclerosis: is decline inevitable? J Neurol. 2020 May;267(5):1464-75.

2 Amato MP, Morra VB, Falautano M, Ghezzi A, Goretti B, Patti F, et al. Cognitive assessment in multiple sclerosis-an Italian consensus. Neurol Sci. 2018 Aug;39(8):1317-24.

3 Damasceno A, Pimentel-Silva LR, Damasceno BP, Cendes F. Cognitive trajectories in relapsing-remitting multiple sclerosis: a longitudinal 6-year study. Mult Scler. 2019 Oct 11; 26(13):1740-51.

4 Silveira C, Guedes R, Maia D, Curral R, Coelho R. Neuropsychiatric symptoms of multiple sclerosis: atate of the art. Psychiatry Investig. 2019 Dec;16(12):877-88.

5 Carotenuto A, Moccia M, Costabile T, Signoriello E, Paolicelli D, Simone M, et al. Associations between cognitive impairment at onset and disability accrual in young people with multiple sclerosis. Sci Rep. 2019 Dec 2;9: 18074.

6 Sachdev PS, Blacker D, Blazer DG, Ganguli $M$, Jeste DV, Paulsen JS, et al. Classifying neurocognitive disorders: the DSM-5 approach. Nat Rev Neurol. 2014 Nov;10(11):634-42.

7 Benedict RH, Amato MP, Boringa J, Brochet B, Foley F, Fredrikson S, et al. Brief international cognitive assessment for MS (BICAMS): international standards for validation. BMC Neurol. 2012 Jul 16;12:55.

8 Beier M, Gromisch ES, Hughes AJ, Alschuler KN, Madathil R, Chiaravalloti N, et al. Proposed cut scores for tests of the brief international cognitive assessment of multiple sclerosis (BICAMS). J Neurol Sci. 2017 Oct 15; 381:110-6.

9 Benedict RH, Pol J, Yasin F, Hojnacki D, Kolb C, Eckert S, et al. Recovery of cognitive function after relapse in multiple sclerosis. Mult Scler. 2020 Jan 23;27(1):71-8.

10 Polman CH, Reingold SC, Banwell B, Clanet M, Cohen JA, Filippi M, et al. Diagnostic criteria for multiple sclerosis: 2010 revisions to the McDonald criteria. Ann Neurol. 2011; 69(2):292-302.

11 Barbarotto R, Laiacona M, Frosio R, Vecchio $\mathrm{M}$, Farinato A, Capitani E. A normative study on visual reaction times and two stroop colour-word tests. Ital J Neurol Sci. 1998;19(3): 161-70.
12 Amodio P, Wenin H, Del Piccolo F, Mapelli D, Montagnese S, Pellegrini A, et al. Variability of trail making test, symbol digit test and line trait test in normal people. A normative study taking into account age-dependent decline and sociobiological variables. Aging Clin Exp Res. 2002 Apr;14(2):117-31.

13 Caffarra P, Vezzadini G, Dieci F, Zonato F, Venneri A. Rey-osterrieth complex figure: normative values in an Italian population sample. Neurol Sci. 2002 Mar;22(6):443-7.

14 Carlesimo GA, Caltagirone C, Gainotti G. The mental deterioration battery: normative data, diagnostic reliability and qualitative analyses of cognitive impairment. The group for the standardization of the mental deterioration battery. Eur Neurol. 1996;36(6):37884.

15 Spaccavento S, Cafforio E, Cellamare F, Colucci A, Di Palma A, Falcone R, et al. Italian adaptation of the functional outcome questionnaire: aphasia: initial psychometric evaluation. Disabil Rehabil. 2018 Dec;40(24): 2925-30.

16 Nichelli $\mathrm{P}$, Leone $\mathrm{M}$, Caronna A, Imbornone E, Alberoni M, Zuffi M, et al. Taratura di un test di stime cognitive di impiego diagnostico in clinica: stime dei tempi e dei pesi (STEP). Nuova Riv Neurol. 2002;12:37-42.

17 Barletta-Rodolfi C, Gasparini F, Ghidoni E. Kit del neuropsicologo Italiano. Bologna, Nvartis: Società Italiana di Neuropsicologia; 2011.

18 Dusankova JB, Kalincik T, Havrdova E, Benedict RH. Cross cultural validation of the minimal assessment of cognitive function in multiple sclerosis (MACFIMS) and the brief international cognitive assessment for multiple sclerosis (BICAMS). Clin Neuropsychol. 2012;26(7):1186.

19 Baetge SJ, Filser M, Renner A, Ullrich S, Lassek C, Penner IK. On the validity of single tests, two-test combinations and the full brief international cognitive assessment for multiple sclerosis (BICAMS) in detecting patients with cognitive impairment. Mult Scler. 2019 Nov 19;26(14):1919-28.
20 Sokolov AA, Grivaz P, Bove R. Cognitive deficits in multiple sclerosis: recent advances in treatment and neurorehabilitation. Curr Treat Options Neurol. 2018 Oct 22;20(12):53.

21 Kappos L, Edan G, Freedman MS, Montalbán $X$, Hartung HP, Hemmer B, et al. The 11-year long-term follow-up study from the randomized BENEFIT CIS trial. Neurology. 2016 Sep 6;87(10):978-87.

22 Mattioli F, Stampatori C, Bemmomi F, Capra R. Natalizumab efficiacy on cognitive impairment in MS. Neurol Sci. 2001;31(Suppl 3): S321-3.

23 Strober L, DeLuca J, Benedict RHB, Jacobs A, Chiaravalloti N, Hudson LD, et al. "Symbol digit modalities test: a valid clinical trial endpoint for measuring cognition in multiple sclerosis". Mult Scler. 2019;25:1781-90.

24 Benedict RH, DeLuca J, Phillips G, LaRocca N, Hudson LD, Rudick R. Validity of the symbol digit modalities test as a cognition performance outcome measure for multiple sclerosis. Mult Scler. 2017 Apr;23(5):721-33.

25 Baetge SJ, Filser M, Renner A, Ullrich S, Lassek C, Penner IK. On the validity of single tests, two-test combinations and the full brief international cognitive assessment for multiple sclerosis (BICAMS) in detecting patients with cognitive impairment. Mult Scler. 2019 Nov 19;26(14):1919-28.

26 Kalb R, Beier M, Benedict RH, Charvet L, Costello K, Feinstein A, et al. Recommendations for cognitive screening and management in multiple sclerosis care. Mult Scler. 2018 Nov;24(13): 1665-80.

27 Benedict RH, Morrow SA, Weinstock Guttman B, Cookfair D, Schretlen DJ. Cognitive reserve moderates decline in information processing speed in multiple sclerosis patients. J Int Neuropsychol Soc. 2010;16(5): 829-35.

28 Ruano L, Portaccio E, Goretti B, Niccolai C, Severo M, Patti F, et al. Age and disability drive cognitive impairment in multiple sclerosis across disease subtypes. Mult Scler. 2017 Aug;23(9):1258-67.

29 Benedict RH, DeLuca J, Phillips G, LaRocca N, Hudson LD, Rudick R. Validity of the symbol digit modalities test as a cognition performance outcome measure for multiple sclerosis. Mult Scler. 2017;23(5):721-33. 